Multi-temperature Euler hydrodynamics for a reacting gas from a kinetic approach to rarefied mixtures with resonant collisions

This content has been downloaded from IOPscience. Please scroll down to see the full text.

2011 EPL 9555002

(http://iopscience.iop.org/0295-5075/95/5/55002)

View the table of contents for this issue, or go to the journal homepage for more

Download details:

IP Address: 159.149.103.9

This content was downloaded on 07/01/2015 at 13:46

Please note that terms and conditions apply. 


\title{
Multi-temperature Euler hydrodynamics for a reacting gas from a kinetic approach to rarefied mixtures with resonant collisions
}

\author{
M. Bisi, G. Martalò and G. SpigA ${ }^{(a)}$ \\ Dept. of Mathematics, Parma University - Viale delle Scienze 53/A, I-43124 Parma, Italy, EU
}

received 31 May 2011; accepted 12 July 2011

published online 10 August 2011

PACS 51.10.+y - Kinetic and transport theory of gases

PACS $47.45 .-n$ - Rarefied gas dynamics

PACS 82.60.-s - Chemical thermodynamics

\begin{abstract}
Starting from a Boltzmann kinetic model for a gas mixture with bimolecular chemical reaction, hydrodynamic equations at Euler level are deduced by a consistent hydrodynamic limit in the presence of resonance, namely when the fast process driving evolution is constituted by elastic scattering between particles of the same species. The structure of the resulting multi-temperature and multi-velocity fluid-dynamic description is briefly commented on, and some results in closed analytical form are given for the special case of Maxwellian collision kernel.
\end{abstract}

Copyright (c) EPLA, 2011

Introduction. - It is well known that, in thermally non-equilibrium conditions, when vibrational relaxation and chemical reactions proceed at the gas-dynamic time scale, a one-temperature gas flow description is not valid, and more rigorous models of kinetics, gas dynamics and transport properties are required [1]. On the other hand, a multi-temperature approach naturally arises when atomic masses of the constituents are different, or in plasmas at high temperature [2], as well as in several problems of aerothermodynamics, like in the flow field around hypersonic vehicles at high altitude [3]. Even though multi-temperature models entail practical difficulties with measuring the temperature of each component, the macroscopic theory of homogeneous mixtures is well developed in the framework of rational thermodynamics [4] on the assumption that each constituent obeys the same balance laws as a single fluid. In this frame, several papers have been published in recent years, and we may quote for instance, without being exhaustive, $[5,6]$. Indeed, the matter seems to attract a broad and intensive attention nowadays, and, as usual, a consistent formal derivation as suitable hydrodynamic limit of a kinetic theory description would be highly desirable [7]. The task is quite uneasy if one wants to include into the picture an essential ingredient like the occurrence of chemical reactions. Such types of interactions involve a rather heavy machinery at the Boltzmann level [8], one can see for instance the kinetic models proposed in [9] and [10], where the

\footnotetext{
(a) E-mail: giampiero.spiga@unipr.it
}

internal molecular structure was taken into account by a discrete or continuous energy variable. The hydrodynamic limit following from the reactive Boltzmann equations depends crucially, of course, on the fast processes driving the evolution, whose collision operators are labeled, upon scaling, by an inverse small parameter (typically, a Knudsen number). In [11] it was shown that, when the dominant operator is made up by all mechanical encounters preserving kinetic energy, including possible "resonant" collisions with change of internal state within the same species, as allowed by some form of degeneracy in the energy levels, hydrodynamic variables (apart from the usual mass densities) turn out to be given by a unique mass velocity and a unique translational temperature for the mixture, plus an internal (typically, vibrational) temperature for each species. The hydrodynamic limit is thus of multitemperature type, and seems to fit formulae of common practical use in physics [11]. The slow gas-dynamic relaxation will lead eventually to equalization of all temperatures, and to a mass action law for chemical equilibrium. A more detailed fluid-dynamic description could involve the presence of two temperatures for each species (one translational and one internal) as well as of single mass velocities. It is just this formidable task that we start addressing here, where, to begin with, we shall confine ourselves to the simplifying assumption that all species are mono-atomic-like (endowed with only translational degrees of freedom). It is assumed that fast resonant collisions driving the overall evolution are constituted by elastic collisions between particles of the same species. 
Still for simplicity, a single reversible bimolecular reaction $A_{1}+A_{2} \leftrightharpoons A_{3}+A_{4}$ is dealt with, and only four participating species are considered. The Euler equations for the 20 hydrodynamic variables (densities, velocities, and temperatures of each component) are derived and briefly discussed. Closed form analytical expressions for the slow collision contributions in the balance equations may be achieved under additional assumptions, like Maxwell collision model or negligible heat of reaction.

Kinetic equations. - The starting point of our analysis is the set of integro-differential Boltzmann-like equations governing the distribution functions of the four participating species, reading, in our assumptions [12],

$$
\frac{\partial f_{i}}{\partial t}+\mathbf{v} \cdot \nabla_{\mathbf{x}} f_{i}=\frac{1}{\varepsilon} I_{i i}\left[f_{i}, f_{i}\right]+\sum_{j \neq i} I_{i j}\left[f_{i}, f_{j}\right]+J_{i}[\mathbf{f}]
$$

where

$$
\begin{aligned}
& \int \varphi_{i}(\mathbf{v}) I_{i j}\left[f_{i}, f_{j}\right](\mathbf{v}) \mathrm{d}_{3} \mathbf{v}=\iiint B_{i j}\left(g, \hat{\mathbf{n}} \cdot \hat{\mathbf{n}}^{\prime}\right) \\
& \times\left[\varphi_{i}\left(\mathbf{v}_{i j}\right)-\varphi_{i}(\mathbf{v})\right] f_{i}(\mathbf{v}) f_{j}(\mathbf{w}) \mathrm{d}_{3} \mathbf{v} \mathrm{d}_{3} \mathbf{w} \mathrm{d}_{2} \hat{\mathbf{n}}^{\prime}
\end{aligned}
$$

is the elastic scattering operator (in weak form) for $(i, j)$ encounters, and

$$
\begin{aligned}
& \int \varphi_{i}(\mathbf{v}) J_{i}[\mathbf{f}](\mathbf{v}) \mathrm{d}_{3} \mathbf{v}=\iiint \varphi_{i}\left(\mathbf{v}_{h k}^{i j}\right) U\left(g^{2}-\delta_{h k}^{i j}\right) \\
& \times\left(\frac{\mu_{i j}}{\mu_{h k}}\right)^{3 / 2}\left(1+\frac{\mu_{i j}}{\mu_{h k}} \frac{\delta_{i j}^{h k}}{g^{2}}\right)^{1 / 2} B_{i j}^{h k}\left(g_{h k}^{i j}, \hat{\mathbf{n}} \cdot \hat{\mathbf{n}}^{\prime}\right) \\
& \times f_{h}(\mathbf{v}) f_{k}(\mathbf{w}) \mathrm{d}_{3} \mathbf{v} \mathrm{d}_{3} \mathbf{w} \mathrm{d}_{2} \hat{\mathbf{n}}^{\prime}-\iiint \varphi_{i}(\mathbf{v}) U\left(g^{2}-\delta_{i j}^{h k}\right) \\
& \times B_{i j}^{h k}\left(g, \hat{\mathbf{n}} \cdot \hat{\mathbf{n}}^{\prime}\right) f_{i}(\mathbf{v}) f_{j}(\mathbf{w}) \mathrm{d}_{3} \mathbf{v} \mathrm{d}_{3} \mathbf{w} \mathrm{d}_{2} \hat{\mathbf{n}}^{\prime}
\end{aligned}
$$

is the reactive (weak) collision integral for species $i$, where the admissible sequences $(i, j, h, k)$ are $(1,2,3,4)$, $(2,1,4,3),(3,4,1,2),(4,3,2,1)$. One can make reference to only one of the reactive collision kernels (relative speed times cross-sections), by virtue of the micro-reversibility condition linking together $B_{i j}^{h k}$ and $B_{h k}^{i j}$, while $B_{i j}=B_{i j}^{i j}$. We set $\mathbf{g}=\mathbf{v}-\mathbf{w}=g \hat{\mathbf{n}}$, with $g=|\mathbf{g}|$, for the relative velocity, $r_{i j}=m_{i} /\left(m_{i}+m_{j}\right)$ for the mass ratios, and $\mu_{i j}=$ $r_{i j} m_{j}$ for the reduced masses.

Post-collision velocities in (2) and (3) read as

$$
\mathbf{v}_{i j}=r_{i j} \mathbf{v}+r_{j i}\left(\mathbf{w}+g \hat{\mathbf{n}}^{\prime}\right), \quad \mathbf{v}_{i j}^{h k}=r_{i j} \mathbf{v}+r_{j i} \mathbf{w}+r_{k h} g_{i j}^{h k} \hat{\mathbf{n}}^{\prime}
$$

and analogous expressions hold for $\mathbf{w}_{i j}$ and $\mathbf{w}_{i j}^{h k}$, where

$$
g_{i j}^{h k}=\left[\frac{\mu_{i j}}{\mu_{h k}}\left(g^{2}-\delta_{i j}^{h k}\right)\right]^{1 / 2}, \quad \delta_{i j}^{h k}=2 \frac{E_{h}+E_{k}-E_{i}-E_{j}}{\mu_{i j}}
$$

with the real number $\delta_{i j}^{h k}$ measuring the transfer of chemical energy into kinetic energy, and is positive or negative according to whether the collision $(i, j) \rightarrow(h, k)$ is endothermic or exothermic. In the former case, the unit step function $U$ in (3) actually imposes a threshold for the reaction to occur. Possible activation energies are accounted for by the reactive kernels $B$. We may always conventionally assume that $\Delta E=E_{3}+E_{4}-E_{1}-E_{2} \geqslant 0$. Finally, the small positive parameter $\varepsilon$ in (1) stands for the proper Knudsen number relevant to the considered problem and represents, for instance, the ratio of the mean scattering collision time for equal species, to any of the other characteristic times, supposed all of the same order of magnitude, and much longer than the previous one. We recall from [9] that collision equilibria for (1) are provided by the seven parameter family of Maxwellians

$$
f_{i}^{M}(\mathbf{v})=n_{i}\left(\frac{m_{i}}{2 \pi T}\right)^{3 / 2} \exp \left[-\frac{m_{i}}{2 T}(\mathbf{v}-\mathbf{u})^{2}\right]
$$

at a common mass velocity $\mathbf{u}$ and temperature $T$, and with number densities related by the classical mass action law

$$
\frac{n_{1} n_{2}}{n_{3} n_{4}}=\left(\frac{\mu_{12}}{\mu_{34}}\right)^{3 / 2} \mathrm{e}^{\Delta E / T} .
$$

Entropy dissipation and stability of equilibria are quantified by the pertinent Lyapunov functional

$$
H[\mathbf{f}]=\sum_{i=1}^{4} \int f_{i} \log \left(\frac{f_{i}}{m_{i}^{3}}\right) \mathrm{d}_{3} \mathbf{v} .
$$

Macroscopic fields for each species are defined in the standard way, and include densities $n_{i}$, mean velocities $\mathbf{u}_{i}$, species temperatures $T_{i}$, which make up, as usual, the macroscopic observables for the gas as a whole, namely $n$, $\rho, \mathbf{u}, T$, while the internal energy density is the sum of the thermal component $\frac{3}{2} n T$ with the chemical contribution $\sum_{i=1}^{4} E_{i} n_{i}$.

We are interested in the hydrodynamic closure of (1) in the asymptotic limit when $\varepsilon \rightarrow 0$, and in particular we shall confine ourselves here to the zeroth-order approximation (Euler level). We are led thus to the investigation of the dominant operator driving the process (1), defined by the vector $\left(I_{11}, I_{22}, I_{33}, I_{44}\right)$. For each of the $I_{i i}$ the standard elastic single species analysis applies, and we end up with a vector of dominant collision equilibria, a 20-parameter family of local Maxwellians

$$
M_{i}(\mathbf{v})=n_{i}\left(\frac{m_{i}}{2 \pi T_{i}}\right)^{3 / 2} \exp \left[-\frac{m_{i}}{2 T_{i}}\left(\mathbf{v}-\mathbf{u}_{i}\right)^{2}\right]
$$

with free parameters $n_{i}, \mathbf{u}_{i}, T_{i}$. This fast part of the collision operator preserves then mass, momentum, and kinetic energy within each single species. Taking weak forms of (1) relevant to such collision invariants, we get a set of 20 macroscopic balance equations for the physical fields, including collision contributions arising from the slow part of the collision operator, since that part prescribes transfer of momentum and kinetic energy from one species to another, as well as transfer of mass among species, and exchange of energy between its kinetic 
and chemical form. In conclusion, the 20 macroscopic "conservation" (for the fast operator) equations read as

$$
\begin{aligned}
& \frac{\partial n_{i}}{\partial t}+\nabla_{\mathbf{x}} \cdot\left(n_{i} \mathbf{u}_{i}\right)=Q_{i}^{\mathrm{ch}}, \\
& \frac{\partial}{\partial t}\left(\rho_{i} \mathbf{u}_{i}\right)+\nabla_{\mathbf{x}} \cdot\left(\rho_{i} \mathbf{u}_{i} \otimes \mathbf{u}_{i}\right)+\nabla_{\mathbf{x}} \cdot \mathbf{P}_{i}=\sum_{j \neq i} \mathbf{R}_{i j}^{\mathrm{me}}+\mathbf{R}_{i}^{\mathrm{ch}}, \\
& \frac{\partial}{\partial t}\left(\frac{1}{2} \rho_{i} u_{i}^{2}+\frac{3}{2} n_{i} T_{i}\right)+\nabla_{\mathbf{x}} \cdot\left[\left(\frac{1}{2} \rho_{i} u_{i}^{2}+\frac{3}{2} n_{i} T_{i}\right) \mathbf{u}_{i}\right. \\
& \left.+\mathbf{P}_{i} \cdot \mathbf{u}_{i}+\mathbf{q}_{i}\right]=\sum_{j \neq i} S_{i j}^{\mathrm{me}}+S_{i}^{\mathrm{ch}}
\end{aligned}
$$

with $\mathbf{P}_{i}$ and $\mathbf{q}_{i}$ for single-species pressure tensor and heat flux, and with mechanical and chemical exchange rates

$$
\begin{aligned}
\mathbf{R}_{i j}^{\mathrm{me}} & =\int m_{i} \mathbf{v} I_{i j} \mathrm{~d}_{3} \mathbf{v}, \quad S_{i j}^{\mathrm{me}}=\int \frac{1}{2} m_{i} v^{2} I_{i j} \mathrm{~d}_{3} \mathbf{v}, \\
Q_{i}^{\mathrm{ch}} & =\int J_{i} \mathrm{~d}_{3} \mathbf{v}, \quad \mathbf{R}_{i}^{\mathrm{ch}}=\int m_{i} \mathbf{v} J_{i} \mathrm{~d}_{3} \mathbf{v}, \\
S_{i}^{\mathrm{ch}} & =\int \frac{1}{2} m_{i} v^{2} J_{i} \mathrm{~d}_{3} \mathbf{v} .
\end{aligned}
$$

It is easy to see that, as physically clear,

$$
Q_{1}^{\mathrm{ch}}=Q_{2}^{\mathrm{ch}}=-Q_{3}^{\mathrm{ch}}=-Q_{4}^{\mathrm{ch}} \equiv Q^{\mathrm{ch}} .
$$

Moreover, due to momentum conservation in each chemical event, we have

$$
\mathbf{R}_{1}^{\mathrm{ch}}+\mathbf{R}_{2}^{\mathrm{ch}}+\mathbf{R}_{3}^{\mathrm{ch}}+\mathbf{R}_{4}^{\mathrm{ch}}=\mathbf{0} .
$$

Finally, due to total energy conservation in each reactive encounter, we get

$$
S_{1}^{\mathrm{ch}}+S_{2}^{\mathrm{ch}}+S_{3}^{\mathrm{ch}}+S_{4}^{\mathrm{ch}}=-Q^{\mathrm{ch}} \Delta E .
$$

In view of the angular integrations involved in (2) and (3) it proves convenient to introduce the angle integrated collision kernels

$$
\begin{aligned}
& B_{i j}^{k}(g)=\int_{S^{2}}\left(\hat{\mathbf{n}} \cdot \hat{\mathbf{n}}^{\prime}\right)^{k} B_{i j}\left(g, \hat{\mathbf{n}} \cdot \hat{\mathbf{n}}^{\prime}\right) \mathrm{d}_{2} \hat{\mathbf{n}}^{\prime}, \quad k=0,1, \\
& \bar{B}_{i j}(g)=B_{i j}^{0}(g)-B_{i j}^{1}(g)
\end{aligned}
$$

with $B_{i j}^{0}>0,\left|B_{i j}^{1}\right|<B_{i j}^{0}$ (we exclude the degenerate cases of delta-like forward or backward scattering), and then $\bar{B}_{i j}>0$. Analogously

$$
B_{k}(g)=\int_{S^{2}}\left(\hat{\mathbf{n}} \cdot \hat{\mathbf{n}}^{\prime}\right)^{k} B_{12}^{34}\left(g, \hat{\mathbf{n}} \cdot \hat{\mathbf{n}}^{\prime}\right) \mathrm{d}_{2} \hat{\mathbf{n}}^{\prime}, \quad k=0,1,
$$

with $B_{0}>0$ and $\left|B_{1}\right|<B_{0}$. In particular we may write

$$
\begin{aligned}
Q^{\mathrm{ch}} & =\left(\frac{\mu_{12}}{\mu_{34}}\right)^{3 / 2} \iint B_{0}\left(g_{34}^{12}\right)\left(1+\frac{2 \Delta E}{\mu_{34} g^{2}}\right)^{1 / 2} \\
& \times f_{3}(\mathbf{v}) f_{4}(\mathbf{w}) \mathrm{d}_{3} \mathbf{v} \mathrm{d}_{3} \mathbf{w} \\
& -\iint B_{0}(g) U\left(g^{2}-\frac{2 \Delta E}{\mu_{12}}\right) f_{1}(\mathbf{v}) f_{2}(\mathbf{w}) \mathrm{d}_{3} \mathbf{v} \mathrm{d}_{3} \mathbf{w}
\end{aligned}
$$

The set (10) is of course exact but not closed, involving higher-order moments and several complicated integrals of the unknown distribution functions. The lowest-order fluid-dynamic closure will be discussed in the next section, though the task of casting all transfer rates in closed analytical form seems hopeless a priori for general collision kernels. Notice that a proper use of (12), (13), (14) into (10) would allow to recover the seven exact conservation equations holding for the present problem, representing conservation of mass in the independent pairs $(1,3),(1,4),(2,4)$, conservation of momentum in the mixture, and conservation of total (mechanical plus chemical) energy in the mixture.

Fluid-dynamic Euler equations. - The sought hydrodynamic closure is achieved by simply substituting into the set (10) the fast collision equilibrium (9) for the actual distribution functions. We get at once $\mathbf{P}_{i}=n_{i} T_{i} \mathbf{I}$, where $\mathbf{I}$ is the identity tensor, and $\mathbf{q}_{i}=\mathbf{0}$ (non-viscous and non-conductive fluid), and moreover also collision contributions $Q, \mathbf{R}, S$ become, in principle, some functions $\hat{Q}, \hat{\mathbf{R}}, \hat{S}$ of the 20 macroscopic fields $n_{i}, \mathbf{u}_{i}, T_{i}$. We rewrite our Euler equations as

$$
\frac{\partial n_{i}}{\partial t}+\nabla_{\mathbf{x}} \cdot\left(n_{i} \mathbf{u}_{i}\right)=\lambda_{i} \hat{Q}^{\mathrm{ch}}
$$

$\frac{\partial}{\partial t}\left(\rho_{i} \mathbf{u}_{i}\right)+\nabla_{\mathbf{x}} \cdot\left(\rho_{i} \mathbf{u}_{i} \otimes \mathbf{u}_{i}\right)+\nabla_{\mathbf{x}}\left(n_{i} T_{i}\right)=\sum_{j \neq i} \hat{\mathbf{R}}_{i j}^{\mathrm{me}}+\hat{\mathbf{R}}_{i}^{\mathrm{ch}}$,

$\frac{\partial}{\partial t}\left(\frac{1}{2} \rho_{i} u_{i}^{2}+\frac{3}{2} n_{i} T_{i}\right)+\nabla_{\mathbf{x}} \cdot\left[\left(\frac{1}{2} \rho_{i} u_{i}^{2}\right.\right.$

$\left.\left.+\frac{5}{2} n_{i} T_{i}\right) \mathbf{u}_{i}\right]=\sum_{j \neq i} \hat{S}_{i j}^{\mathrm{me}}+\hat{S}_{i}^{\mathrm{ch}}$

where $\lambda_{i}$ is the $i$-th stoichiometric coefficient $\left(\lambda_{1}=\lambda_{2}=\right.$ $\left.1=-\lambda_{3}=-\lambda_{4}\right)$. The product of the two Maxwellians at different velocities and temperatures may be cast as

$$
\begin{aligned}
& M_{i}(\mathbf{v}) M_{j}(\mathbf{w})=n_{i} n_{j}\left(\frac{m_{i}}{2 \pi T_{i}}\right)^{3 / 2}\left(\frac{m_{j}}{2 \pi T_{j}}\right)^{3 / 2} \\
& \times \exp \left[-\alpha_{i j}\left(\mathbf{G}_{i j}+\gamma_{i j} \mathbf{g}-\boldsymbol{\delta}_{i j}\right)^{2}\right] \mathrm{e}^{-\beta_{i j}\left[\mathbf{g}-\left(\mathbf{u}_{i}-\mathbf{u}_{j}\right)\right]^{2}}
\end{aligned}
$$

with $\mathbf{G}_{i j}=r_{i j} \mathbf{v}+r_{j i} \mathbf{w}$, and

$$
\begin{aligned}
& \alpha_{i j}=\frac{m_{i}}{2 T_{i}}+\frac{m_{j}}{2 T_{j}}, \quad \beta_{i j}=\left(\frac{2 T_{i}}{m_{i}}+\frac{2 T_{j}}{m_{j}}\right)^{-1} \\
& \gamma_{i j}=\frac{\mu_{i j}}{\alpha_{i j}}\left(\frac{1}{2 T_{i}}-\frac{1}{2 T_{j}}\right), \boldsymbol{\delta}_{i j}=\frac{1}{\alpha_{i j}}\left(\frac{m_{i}}{2 T_{i}} \mathbf{u}_{i}+\frac{m_{j}}{2 T_{j}} \mathbf{u}_{j}\right) .
\end{aligned}
$$

Due to this heavy, but convenient, form, integrations in all collision contributions may be performed in terms of center of mass and relative velocities. It is easy to see first that $\hat{\mathbf{R}}_{i j}^{\text {me }}$ vanishes when $\mathbf{u}_{i}=\mathbf{u}_{j}$, and that, for $\mathbf{u}_{i} \neq \mathbf{u}_{j}$, it is parallel to the vector $\mathbf{u}_{i}-\mathbf{u}_{j}$. It proves thus convenient 
introducing Gaussian averaged collision kernels $\left(\mathbf{u}_{i} \neq \mathbf{u}_{j}\right)$

$$
\left\langle\bar{B}_{i j}\right\rangle=\frac{\int \bar{B}_{i j}(g) \mathbf{g} \cdot\left(\mathbf{u}_{i}-\mathbf{u}_{j}\right) \mathrm{e}^{-\beta_{i j}\left[\mathbf{g}-\left(\mathbf{u}_{i}-\mathbf{u}_{j}\right)\right]^{2}} \mathrm{~d}_{3} \mathbf{g}}{\int \mathbf{g} \cdot\left(\mathbf{u}_{i}-\mathbf{u}_{j}\right) \mathrm{e}^{-\beta_{i j}\left[\mathbf{g}-\left(\mathbf{u}_{i}-\mathbf{u}_{j}\right)\right]^{2}} \mathrm{~d}_{3} \mathbf{g}},
$$

so that one ends up with the convenient representation

$$
\hat{\mathbf{R}}_{i j}^{\mathrm{me}}\left(n_{i}, \mathbf{u}_{i}, T_{i}, n_{j}, \mathbf{u}_{j}, T_{j}\right)=-\mu_{i j} n_{i} n_{j}\left\langle\bar{B}_{i j}\right\rangle\left(\mathbf{u}_{i}-\mathbf{u}_{j}\right),
$$

where $\left\langle\bar{B}_{i j}\right\rangle$ is a function of $\left|\mathbf{u}_{i}-\mathbf{u}_{j}\right|, T_{i}$, and $T_{j}$. The simplest collision model, as usual in kinetic theory, is provided by the Maxwell molecules, in which case $\bar{B}_{i j}=$ constant $=\left\langle\bar{B}_{i j}\right\rangle$, and indeed (22) would follow straightforwardly from its definition. It is remarkable that in this assumption the result (22) is exact, and not only asymptotic (i.e., $\mathbf{R}_{i j}^{\mathrm{me}}=\hat{\mathbf{R}}_{i j}^{\mathrm{me}}$ ), since it may be deduced without any need of replacing the actual distribution functions $f_{i}$ by the corresponding local Maxwellians $M_{i}$. Just as an example, we report here on the more realistic model of rigid spheres, $\bar{B}_{i j}(g)=\eta_{i j} g$, for which a lengthy but standard manipulation yields

$$
\begin{aligned}
\left\langle\bar{B}_{i j}\right\rangle= & \frac{\eta_{i j}}{\left(\pi \beta_{i j}\right)^{1 / 2}}\left[\left(\beta_{i j}^{1 / 2}\left|\mathbf{u}_{i}-\mathbf{u}_{j}\right|+\frac{1}{\beta_{i j}^{1 / 2}\left|\mathbf{u}_{i}-\mathbf{u}_{j}\right|}\right.\right. \\
& \left.-\frac{1}{4 \beta_{i j}^{3 / 2}\left|\mathbf{u}_{i}-\mathbf{u}_{j}\right|^{3}}\right) \sqrt{\pi} \operatorname{erf}\left(\beta_{i j}^{1 / 2}\left|\mathbf{u}_{i}-\mathbf{u}_{j}\right|\right) \\
& \left.+\left(1+\frac{1}{2 \beta_{i j}\left|\mathbf{u}_{i}-\mathbf{u}_{j}\right|^{2}}\right) \mathrm{e}^{-\beta_{i j}\left|\mathbf{u}_{i}-\mathbf{u}_{j}\right|^{2}}\right]
\end{aligned}
$$

where erf denotes error function, and with limiting trends

$$
\begin{aligned}
& \left\langle\bar{B}_{i j}\right\rangle=\frac{8}{3 \sqrt{\pi}} \frac{\eta_{i j}}{\beta_{i j}^{1 / 2}}+O\left(\left|\mathbf{u}_{i}-\mathbf{u}_{j}\right|^{2}\right) \text { for }\left|\mathbf{u}_{i}-\mathbf{u}_{j}\right| \rightarrow 0 \\
& \left\langle\bar{B}_{i j}\right\rangle \sim \eta_{i j}\left|\mathbf{u}_{i}-\mathbf{u}_{j}\right| \text { for }\left|\mathbf{u}_{i}-\mathbf{u}_{j}\right| \rightarrow \infty
\end{aligned}
$$

For the mechanical exchange rate of kinetic energy, resorting again to (19), and skipping intermediate details, another, differently averaged, collision kernel

$$
\left\langle\left\langle\bar{B}_{i j}\right\rangle\right\rangle=\frac{\int \bar{B}_{i j}(g) g^{2} \mathrm{e}^{-\beta_{i j}\left[\mathbf{g}-\left(\mathbf{u}_{i}-\mathbf{u}_{j}\right)\right]^{2}} \mathrm{~d}_{3} \mathbf{g}}{\int g^{2} \mathrm{e}^{-\beta_{i j}\left[\mathbf{g}-\left(\mathbf{u}_{i}-\mathbf{u}_{j}\right)\right]^{2}} \mathrm{~d}_{3} \mathbf{g}}
$$

shows up, depending again, in general, on $\left|\mathbf{u}_{i}-\mathbf{u}_{j}\right|, T_{i}$, and $T_{j}$. In conclusion, we may write

$$
\begin{aligned}
& \hat{S}_{i j}^{\mathrm{me}}\left(n_{i}, \mathbf{u}_{i}, T_{i}, n_{j}, \mathbf{u}_{j}, T_{j}\right)=-\mu_{i j} n_{i} n_{j}\left\{\left[\left\langle\bar{B}_{i j}\right\rangle \boldsymbol{\delta}_{i j}\right.\right. \\
& \left.\left.-\gamma_{i j}\left\langle\left\langle\bar{B}_{i j}\right\rangle\right\rangle\left(\mathbf{u}_{i}-\mathbf{u}_{j}\right)\right] \cdot\left(\mathbf{u}_{i}-\mathbf{u}_{j}\right)+\left\langle\left\langle\bar{B}_{i j}\right\rangle\right\rangle \frac{3\left(T_{i}-T_{j}\right)}{m_{i}+m_{j}}\right\}
\end{aligned}
$$

vanishing when both $\mathbf{u}_{i}=\mathbf{u}_{j}$ and $T_{i}=T_{j}$. Once more, a great simplification occurs for Maxwell molecules, since
$\left\langle\bar{B}_{i j}\right\rangle=\left\langle\left\langle\bar{B}_{i j}\right\rangle\right\rangle=\bar{B}_{i j}=$ const, so that

$$
\begin{aligned}
\hat{S}_{i j}^{\mathrm{me}}= & -\mu_{i j} n_{i} n_{j} \bar{B}_{i j}\left[\left(r_{i j} \mathbf{u}_{i}+r_{j i} \mathbf{u}_{j}\right) \cdot\left(\mathbf{u}_{i}-\mathbf{u}_{j}\right)\right. \\
& \left.+\frac{3\left(T_{i}-T_{j}\right)}{m_{i}+m_{j}}\right]
\end{aligned}
$$

and again this expression turns out to be the exact one in such a frame, no need of approximating $f_{i}$ by $M_{i}$.

At this stage, all mechanical (slow) collision contributions in the Euler equations (18) for the collision dominated resonant regime are known in principle (for given collision kernels $B$ ) via eqs. (22) and (26) in terms of the chosen observable fields $n_{i}, \mathbf{u}_{i}, T_{i}$. Analogous steps for the chemical collision contributions might be performed in similar way, with severe additional technical difficulties, as apparent from eqs. (3) and (17). Even upon introducing suitable averages for the chemical collision kernels, the remaining integrals turn out not to be amenable to any of the most common transcendental or special functions. The simplest integration concerns the loss part of $\hat{Q}^{\text {ch }}$, which, after some manipulations, even under Maxwell molecule assumption $\left(B_{0}(g)=\right.$ const), takes a quite huge expression (not given here for brevity) involving Gaussians and error functions with arguments $\left|\mathbf{u}_{1}-\mathbf{u}_{2}\right| \pm \sqrt{2 \Delta E / \mu_{12}}$. The situation becomes worse when trying to calculate $\hat{Q}_{+}^{\text {ch }}$. One realizes that only under quite restrictive assumptions a reasonable analytical form can be achieved. For instance, for Maxwell-like molecules and all equal velocities (multitemperature Euler gas with no diffusion) one can write

$$
\begin{aligned}
& \hat{Q}^{\mathrm{ch}}(\underset{\sim}{n}, \underset{\sim}{T})=\frac{2}{\sqrt{\pi}} B_{0}\left[n_{3} n_{4}\left(\frac{\mu_{12}}{\mu_{34}}\right)^{3 / 2} \mathrm{e}^{\frac{\Delta E}{r_{43} T_{3}+r_{34} T_{4}}}\right. \\
& \left.\times \Gamma\left(\frac{3}{2}, \frac{\Delta E}{r_{43} T_{3}+r_{34} T_{4}}\right)-n_{1} n_{2} \Gamma\left(\frac{3}{2}, \frac{\Delta E}{r_{21} T_{1}+r_{12} T_{2}}\right)\right],
\end{aligned}
$$

where $\Gamma$ denotes an incomplete Euler gamma-function.

For these reasons, in order to get some insight into the Euler description of the reactive events, and to test the reliability of the set (18) as a fluid-dynamic model of the actual evolution problem, we prefer to restrict ourselves here to a chemical collision model as simple as possible, allowing for an analytical explicit expression (still uneasy, but at least manageable) for the collision contributions. We hope to be able to undertake, in future investigations, a more systematic (and, necessarily, computer aided) campaign for more realistic interaction laws. The chemical collision model adopted here is in some sense equivalent to the Maxwellian models for mechanical encounters. It consists in taking Maxwell-like collision kernel $B_{12}^{34}$ depending only on $\hat{\mathbf{n}} \cdot \hat{\mathbf{n}}^{\prime}$, so that its angular moments are constant, and in assuming negligible heat of reaction, so that we may reasonably suppose $\Delta E=0$. Due to micro-reversibility [12], this implies that also the reverse kernel $B_{34}^{12}$ is of Maxwell type. Under the above restriction 
we shall be able to cast all chemical source contributions in closed analytical form, and, moreover, such contributions will be exact, namely holding for the actual distribution functions $f_{i}$ and not only for their asymptotic limits $M_{i}$. In other words, the space homogeneous version of the balance equations (18) is a closed exact set of 20 ordinary differential equations for 20 hydrodynamic variables $n_{i}$, $\mathbf{u}_{i}, T_{i}$.

In the frame of the chemical Maxwellian model described above, the chemical exchange rates in the continuity equations are simply given by

$$
\begin{aligned}
\hat{Q}_{i}^{\mathrm{ch}} & =\lambda_{i} \hat{Q}^{\mathrm{ch}}=\lambda_{i} B_{0}\left[\left(\frac{\mu_{12}}{\mu_{34}}\right)^{3 / 2} n_{3} n_{4}-n_{1} n_{2}\right] \\
& =B_{0}\left[\left(\frac{\mu_{12}}{\mu_{34}}\right)^{\frac{3}{4}\left(1+\lambda_{i}\right)} n_{h} n_{k}-\left(\frac{\mu_{12}}{\mu_{34}}\right)^{\frac{3}{4}\left(1-\lambda_{i}\right)} n_{i} n_{j}\right],
\end{aligned}
$$

where $\quad(i, j, h, k)=(1,2,3,4), \quad(2,1,4,3), \quad(3,4,1,2)$, $(4,3,2,1)$. Taking advantage of the simpler expressions of post-collision velocities, also the first- and second-order power moments of the chemical collision operators are amenable to the first- and second-order moments of the distribution functions, and thus also the quantities $\hat{\mathbf{R}}_{i}^{\mathrm{ch}}$ and $\hat{S}_{i}^{\text {ch }}$ can be calculated explicitly. Skipping technical details, the result reads as

$$
\begin{aligned}
\hat{\mathbf{R}}_{i}^{\mathrm{ch}}= & B_{0}\left(\frac{\mu_{12}}{\mu_{34}}\right)^{\frac{3}{4}\left(1+\lambda_{i}\right)} n_{h} n_{k} r_{i j}\left(m_{h} \mathbf{u}_{h}+m_{k} \mathbf{u}_{k}\right) \\
& -B_{0}\left(\frac{\mu_{12}}{\mu_{34}}\right)^{\frac{3}{4}\left(1-\lambda_{i}\right)} n_{i} n_{j} m_{i} \mathbf{u}_{i} \\
& +B_{1}\left(\frac{\mu_{12}}{\mu_{34}}\right)^{\frac{3+5 \lambda_{i}}{4}} n_{h} n_{k} \mu_{h k}\left(\mathbf{u}_{h}-\mathbf{u}_{k}\right)
\end{aligned}
$$

fulfilling (13), and

$$
\begin{aligned}
\hat{S}_{i}^{\mathrm{ch}}= & B_{0}\left(\frac{\mu_{12}}{\mu_{34}}\right)^{\frac{3}{4}\left(1+\lambda_{i}\right)} n_{h} n_{k}\left[\frac{1}{2} m_{i}\left(r_{h k} \mathbf{u}_{h}+r_{k h} \mathbf{u}_{k}\right)^{2}\right. \\
& +\frac{1}{2} r_{j i} \mu_{h k}\left(\mathbf{u}_{h}-\mathbf{u}_{k}\right)^{2}+\frac{3}{2} r_{i j}\left(r_{h k} T_{h}+r_{k h} T_{k}\right) \\
& \left.+\frac{3}{2} r_{j i}\left(r_{k h} T_{h}+r_{h k} T_{k}\right)\right]-B_{0}\left(\frac{\mu_{12}}{\mu_{34}}\right)^{\frac{3}{4}\left(1-\lambda_{i}\right)} n_{i} n_{j} \\
& \times\left(\frac{1}{2} m_{i} u_{i}^{2}+\frac{3}{2} T_{i}\right)+B_{1}\left(\frac{\mu_{12}}{\mu_{34}}\right)^{\frac{3+5 \lambda_{i}}{4}} n_{h} n_{k} \mu_{h k} \\
& \times\left[\left(r_{h k} \mathbf{u}_{h}+r_{k h} \mathbf{u}_{k}\right) \cdot\left(\mathbf{u}_{h}-\mathbf{u}_{k}\right)\right. \\
& \left.+\frac{3}{m_{h}+m_{k}}\left(T_{h}-T_{k}\right)\right]
\end{aligned}
$$

fulfilling (14), with $\Delta E=0$.

It can be noticed that the full collision equilibrium (6), (7) implies now all equal velocities and temperatures, and a mass action law for densities $n_{1} n_{2}=n_{3} n_{4}\left(\mu_{12} / \mu_{34}\right)^{3 / 2}$.
Of course, it is easy to check that, under such circumstances, all collision contributions $\hat{\mathbf{R}}_{i j}^{\mathrm{me}}, \hat{S}_{i j}^{\mathrm{me}}, \hat{Q}^{\mathrm{ch}}, \hat{\mathbf{R}}_{i}^{\mathrm{ch}}$, $\hat{S}_{i}^{\text {ch }}$ in (18) vanish, as follows from (22), (27), (29), (30), (31).

\section{Explicit results for the Maxwellian model. -} We now investigate in some more detail the simplified problem in which all interactions are fully of Maxwell type, dropping all "me" and "ch" superscripts or subscripts, as well as all hats. The phase space for (18) is 20-dimensional, and initial conditions will be labelled by a 0 superscript. In space homogeneous conditions, due to the conservation laws of the slow collision operator, seven independent first integrals are in order, namely

$$
\begin{aligned}
& n_{i}+n_{j}=n_{i}^{0}+n_{j}^{0}, \quad(i, j)=(1,3),(1,4),(2,4), \\
& \mathbf{u}=\frac{1}{\rho^{0}} \sum_{i=1}^{4} \rho_{i} \mathbf{u}_{i}=\mathbf{u}^{0} \\
& T=\frac{1}{3 n^{0}} \sum_{i=1}^{4} \rho_{i}\left(\mathbf{u}_{i}-\mathbf{u}^{0}\right)^{2}+\frac{1}{n^{0}} \sum_{i=1}^{4} n_{i} T_{i}=T^{0}
\end{aligned}
$$

so that evolution actually takes place in a 13-dimensional subspace, once initial conditions are given, and independent variables may be chosen, for instance, as $n_{1}, \mathbf{u}_{2}, \mathbf{u}_{3}$, $\mathbf{u}_{4}, T_{2}, T_{3}, T_{4}$. In particular, also $n=n^{0}$ and $\rho=\rho^{0}$. As discussed above, the collision operator in (18) vanishes at the "physical" equilibrium $\mathbf{u}_{1}=\mathbf{u}_{2}=\mathbf{u}_{3}=\mathbf{u}_{4}(=\mathbf{u})$, $T_{1}=T_{2}=T_{3}=T_{4}(=T)$, and

$$
\frac{n_{1} n_{2}}{n_{3} n_{4}}=\left(\frac{\mu_{12}}{\mu_{34}}\right)^{\frac{3}{2}} \equiv \xi
$$

(a 7 parameter family with a single velocity and a single temperature, plus mass action law for densities). Indeed, it can be proved that initial conditions determine uniquely an element in the above family, or, in other words, there is a unique element belonging both to that 7-dimensional subspace and to the 13-dimensional subspace of evolution determined by an assigned initial state. In fact, if star denotes equilibrium, it is readily seen that

$$
\mathbf{u}_{1}^{*}=\mathbf{u}_{2}^{*}=\mathbf{u}_{3}^{*}=\mathbf{u}_{4}^{*}=\mathbf{u}^{0}, \quad T_{1}^{*}=T_{2}^{*}=T_{3}^{*}=T_{4}^{*}=T^{0},
$$

and then, after suitable manipulations putting together the first of (32) with (33), one gets for densities a quadratic equation with only one admissible (i.e., positive) solution, namely

$$
\begin{aligned}
n_{1}^{*}= & \frac{1}{2(1-\xi)}\left\{\left[\left(\xi\left(2 n_{1}^{0}+n_{3}^{0}+n_{4}^{0}\right)-\left(n_{1}^{0}-n_{2}^{0}\right)\right)^{2}\right.\right. \\
& \left.+4 \xi(1-\xi)\left(n_{1}^{0}+n_{3}^{0}\right)\left(n_{1}^{0}+n_{4}^{0}\right)\right]^{1 / 2} \\
& \left.-\left[\xi\left(2 n_{1}^{0}+n_{3}^{0}+n_{4}^{0}\right)-\left(n_{1}^{0}-n_{2}^{0}\right)\right]\right\},
\end{aligned}
$$

with $n_{2}^{*}=n_{1}^{*}-\left(n_{1}^{0}-n_{2}^{0}\right), \quad n_{3}^{*}=n_{1}^{0}+n_{3}^{0}-n_{1}^{*}, \quad n_{4}^{*}=n_{1}^{0}+n_{4}^{0}-$ $n_{1}^{*}$. In the special (singular) case $\xi=1$ we would have simply $n_{1}^{*}=\left(n_{1}^{0}+n_{3}^{0}\right)\left(n_{1}^{0}+n_{4}^{0}\right) / n^{0}$ instead of (35). In the 
limiting case $\xi \rightarrow 0^{+}$, the smaller between $n_{1}^{*}$ and $n_{2}^{*}$ tends to zero, whilst, in the opposite limiting case $\xi \rightarrow+\infty$, the smaller between $n_{3}^{*}$ and $n_{4}^{*}$ tends to zero.

We can verify that the fluid-dynamic model (18) does not exhibit spurious collision equilibria, different from (33). To this end, we first notice that setting $Q_{i}=0$ yields a single constraint, the mass action law (33). On using this result into the second of (18) one ends up, after some algebra, with the linear homogeneous algebraic system

$$
\sum_{l=1}^{4} A_{i l} \mathbf{u}_{l}=\mathbf{0}, \quad A_{i l}=\bar{A}_{i l}+A_{i l}^{(0)}+A_{i l}^{(1)},
$$

where $\bar{A}_{i l}$ come from the mechanical interactions, $A_{i l}^{(0)}$ and $A_{i l}^{(1)}$ from the chemical ones relevant to $B_{0}$ and $B_{1}$ (their explicit forms are omitted for brevity). It is easy to see that all matrices $\underset{\sim}{\overline{\mathbf{A}}}, \underset{\sim}{\mathbf{A}^{(0)}}, \underset{\sim}{\mathbf{A}^{(1)}}$ (and then A) are symmetric and singular (sums of rows/columns equal to zero), and that the algebraic system admits at least $\infty^{1}$ non-trivial solutions, proportional to the vector $(1,1,1,1)^{T}$, and corresponding to all equal velocities. We take then $3 \times 3$ sub-matrices of $\underset{\sim}{\overline{\mathbf{A}}}, \underset{\sim}{\mathbf{A}^{(0)}},{\underset{\sim}{\mathbf{A}}}^{(1)}$ made up by the first three rows and columns and label them with a prime. It can be checked by direct computation that $\stackrel{\sim}{\mathbf{A}}^{\prime}$ is strictly diagonally dominant (thus non-singular) and negative definite. A bit more involved manipulation shows that, irrespective of the sign of $B_{1}$, the $3 \times 3$ matrix $\underline{\mathbf{A}}^{\prime(0)}+\underline{\mathbf{A}}^{\prime(1)}$ has a negative determinant and is negative definite as well. Therefore the rank of the overall matrix $\underset{\sim}{\mathbf{A}}$ is equal to 3 , since its sub-matrix made up by the first three rows and columns is negative definite and non-singular, so that the algebraic system (36) does not admit any other solution than the one corresponding to all equal velocities.

Using further the above results on densities and velocities into the third of (18) leads again, after some algebra, to the linear homogeneous algebraic system

$$
\sum_{l=1}^{4} C_{i l} T_{l}=0, \quad C_{i l}=\bar{C}_{i l}+C_{i l}^{(0)}+C_{i l}^{(1)},
$$

where, once more, explicit expressions are omitted for brevity. Matrix $\underset{\mathbf{C}}{\mathbf{C}}$ is symmetric and singular in the same way as matrix $\underset{\sim}{\mathbf{A}}$, and admits again at least $\infty^{1}$ non-trivial solutions, proportional to the vector $(1,1,1,1)^{T}$, corresponding to all equal temperatures. Matrix $\underset{\sim}{\mathbf{C}}$ has exactly the same characteristics as matrix $\underset{\sim}{\mathbf{A}}$ concerning negativity and diagonal dominance of its sub-matrices, and analogously for negativity of the $3 \times 3$ sub-matrix of

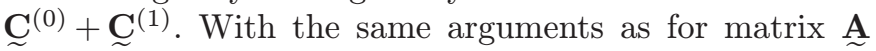
one can conclude that the rank of matrix $\underset{\mathbb{C}}{\mathbf{C}}$ is equal to 3 , and that the algebraic system (37) can not admit non-trivial solutions independent of $(1,1,1,1)^{T}$. This completes the proof that the fluid-dynamic equations (18) admit only the physical collision equilibria, namely the projection of those predicted at the kinetic level, provided by eqs. (34) and (35).

Another robustness test for the present hydrodynamic model concerns entropy dissipation and relaxation to equilibrium. We consider the restriction of the reactive $H$-functional (8) to the finite-dimensional subspace of distribution functions defined by the fast collision equilibria $M_{i}(9)$. This yields a (tentative) Lyapunov function of the kind

$$
\hat{H}=\sum_{i=1}^{4} n_{i}\left[\log \left(\frac{n_{i}}{m_{i}^{3}}\right)+\frac{3}{2} \log \left(\frac{m_{i}}{2 \pi T_{i}}\right)\right],
$$

actually depending, in space homogeneous conditions, on the 13 field variables $n_{1}, \mathbf{u}_{2}, \mathbf{u}_{3}, \mathbf{u}_{4}, T_{2}, T_{3}, T_{4}$, and attaining its minimum at the equilibrium (34), (35). In the space-independent case, formal derivation from (38) yields

$$
\dot{\hat{H}}=\sum_{i=1}^{4} \dot{n}_{i}\left[\log \left(\frac{n_{i}}{m_{i}^{3}}\right)+\frac{3}{2} \log \left(\frac{m_{i}}{2 \pi T_{i}}\right)\right]-\frac{3}{2} \sum_{i=1}^{4} n_{i} \frac{\dot{T}_{i}}{T_{i}}
$$

and, at least under the Maxwellian assumptions of the present section, it is possible to prove, after a very careful and patient calculation, that actually (39) turns out to be negative definite with respect to the unique equilibrium determined by initial conditions.

Work on the uneasy extension of the present results to more realistic collision models and reactions is in progress.

Work performed in the frame of activities sponsored by INdAM-GNFM and by the University of Parma.

\section{REFERENCES}

[1] Kustova E. V. and Nagnibeda E. A., Chem. Phys., 321 (2006) 293.

[2] Bose T. K., High Temperature Gas Dynamics (Springer, Berlin) 2003.

[3] PARK C., Nonequilibrium Hypersonic Aerothermodynamics (Wiley, New York) 1990.

[4] Müller I. and Ruggeri T., Rational Extended Thermodynamics (Springer Verlag, New York) 1998.

[5] Ruggeri T. and Simic S., Math. Methods Appl. Sci., 30 (2007) 827.

[6] Ruggeri T. and Lou J., Phys. Lett. A, 373 (2009) 3052.

[7] Cercignani C., Rarefied Gas Dynamics. From Basic Concepts to Actual Calculations (Cambridge University Press, Cambridge) 2000.

[8] Giovangigli V., Multicomponent Flow Modeling (Birkhäuser, Boston) 1999.

[9] Groppi M. and Spiga G., J. Math. Chem., 26 (1999) 197.

[10] Desvillettes L., Monaco R. and Salvarani F., Eur. J. Mech. B Fluids, 24 (2005) 219.

[11] Groppi M., Spiga G. and Zus F., Phys. Fluids, 18 (2006) 057105.

[12] Bisi M., Groppi M. and Spiga G., Kinetic Methods for Nonconservative and Reacting Systems, edited by Toscani G., in Quaderni di Matematica, Vol. 16 (Aracne Editrice, Roma) 2005, p. 1. 